\title{
INTERNAL SOLITARY WAVES WITH STRATIFICATION IN DENSITY
}

\author{
HUYUN SHA and J.-M. VANDEN-BROECK ${ }^{1}$
}

(Received 3 January 1995; revised 25 May 1995)

\begin{abstract}
Long periodic waves propagating in a closed channel are considered. The fluid consists of two layers of constant densities separated by a layer in which the density varies continuously. The numerical results of Vanden-Broeck and Turner [8] are extended. It is shown that their solutions are particular members of a family of solutions. Solutions are selected by requiring that the streamfunction takes values on the upper and lower walls which are consistent with a uniform stream far upstream. The new solutions are qualitatively similar to those of Vanden-Broeck and Turner [8]. In particular, there are periodic waves characterized by a train of ripples at their troughs. It is shown numerically that these waves approach solitary waves with oscillatory tails as their wavelength increases. Moreover special solutions for which the amplitude of the ripples is almost zero are identified. Such solutions without ripples were previously found for solitary waves with surface tension.
\end{abstract}

\section{Introduction}

The familiar gravity solitary wave on the surface of a fluid in water of finite depth is characterized by a uniform stream in the far field. When a small amount of surface tension is introduced, the solitary wave takes the form of a single crest with, on either side, a small-amplitude oscillation extending to infinity without decrement in its amplitude. Such waves are sometimes called "generalized solitary waves" to distinguish them from the familiar solitary waves. Hunter and Vanden-Broeck [3] calculated long periodic gravity capillary waves with oscillations at their troughs. Vanden-Broeck [7] extended the numerical results of Hunter and Vanden-Broeck [3]. He showed numerically that the periodic waves of Hunter and Vanden-Broeck [3] approach generalized solitary waves as their wavelength is increased. In addition he identified special solutions for which the amplitude of the ripples is essentially zero.

\footnotetext{
'Department of Mathematics and Center for the Mathematical Sciences, University of WisconsinMadison, WI 53706, U.S.A.

(C) Australian Mathematical Society, 1997, Serial-fee code 0334-2700/97
} 
Beale [2] and Sun [5] proved analytically the existence of generalized gravity capillary solitary waves.

Akylas and Grimshaw [1] and Sun and Shen [6] considered solitary internal waves in a density-stratified fluid of shallow depth. By using asymptotics beyond any order, they showed that solitary waves of mode $n>1$ are generalized solitary waves (that is, they have oscillatory tails of infinite extent, consisting of lower-mode short waves). Vanden-Broeck and Turner [8], in an independent work, considered long periodic internal waves. By using a finite difference method in the layer of variable density and boundary integral equation methods in the layers of constant densities, they computed periodic waves of mode 2 whose wavelength is large compared to the thickness of the layer of variable density. They found that these waves are characterized by a train of periodic waves of mode 1 in their troughs. Since long periodic waves can be used as an approximation for solitary waves, their findings suggest the existence of generalized solitary waves for internal waves.

In this paper we consider further the long periodic internal waves of Vanden-Broeck and Turner [8]. We show that the solutions of Vanden-Broeck and Turner [8] are particular members of a family of solutions for which the streamfunction can take any prescribed value on the upper wall. Among this family, the most interesting solution is the one for which the streamfunction takes values on the upper wall (and also on the lower wall) which are consistent with a uniform stream far upstream. Therefore we present a modified scheme in which these conditions on the streamfunction are imposed. Our numerical solutions are qualitatively similar to those of Vanden-Broeck and Turner [8], that is, there are long periodic waves with a train of short waves in their troughs. One main difference is that the new solutions approach a uniform stream as their amplitude decreases (the solutions of Vanden-Broeck and Turner approach conjugate flows as their amplitude decreases). We use a procedure similar to the one developed by Vanden-Broeck [7] to show that the long periodic waves approach generalized solitary waves as their wavelength is increased. More precisely we show that starting with a given periodic wave we can generate a new one by increasing its wavelength by a multiple of the wavelength of the ripples in the trough, leaving the main crest almost intact. By taking larger and larger multiples of the wavelength of the ripples, we can generate longer and longer periodic waves. In the limit this gives a generalized internal solitary wave. We show that the ripples are consistent with linear theory. Finally we identify special solutions for which the amplitude of the ripples is almost zero. Such solutions were not computed by Vanden-Broeck and Turner [8].

The numerical procedure is described in Section 2 and the results are presented in Section 3. 


\section{Formulation}

In this section we summarize the formulation of the problem. More details can be found in Vanden-Broeck and Turner [8].

We consider a periodic wave moving to the left at a constant velocity $\mathrm{c}$ in a fluid bounded above and below by horizontal walls (see Figure 1). The fluid consists of two layers of constant densities $\rho_{1}>\rho_{2}$ separated by a region of linear stratification in density. We assume that the fluid is inviscid, incompressible, and nondiffusive. We choose Cartesian coordinates moving with the wave so that the whole flow is steady. The $x$ axis is on the horizontal bottom and the $y$ axis passes through a crest of the wave. We also assume that the acceleration of gravity $g$ acts in the negative $y$ direction.
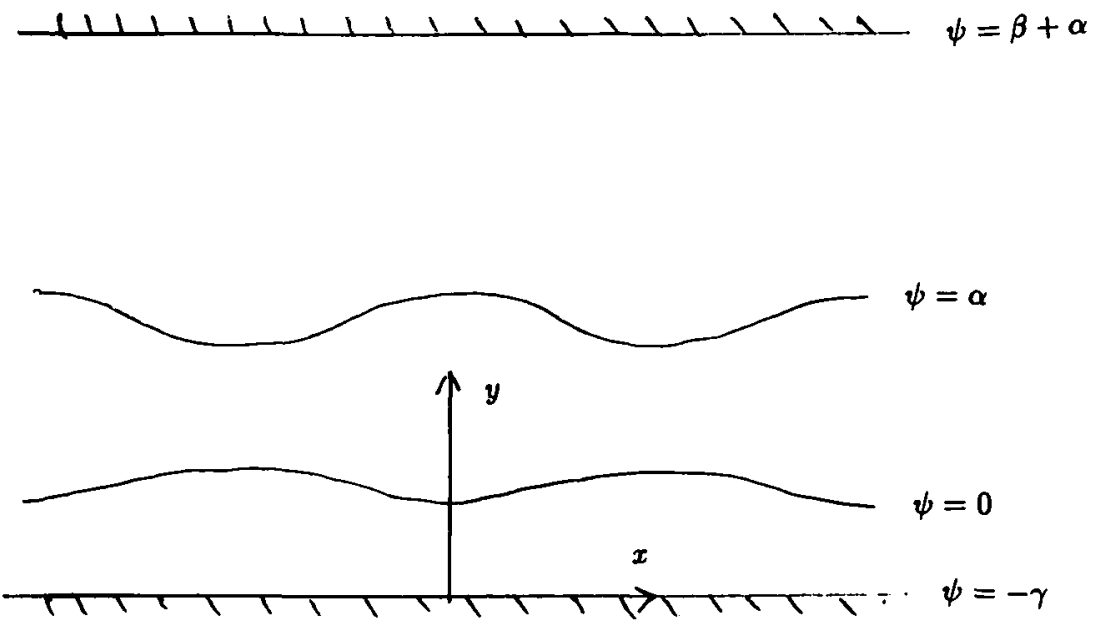

FIGURE 1. Sketch of the wave and of the coordinates.

We denote by $u$ and $v$ the $x$ and $y$ components of the velocity, and by $\psi(x, y)$ the streamfunction, so that

$$
\begin{aligned}
& u=\psi_{y}, \\
& v=-\psi_{x} .
\end{aligned}
$$

Let $\psi=0$ and $\psi=\alpha$ be the two streamlines bounding the region in which the density varies linearly with $\psi$, and let $-\gamma$ and $\alpha+\beta$ be the values of $\psi$ on the lower and upper horizontal walls, respectively. Then, we have the following density distribution

$$
\rho= \begin{cases}\rho_{1}, & \text { when }-\gamma<\psi<0 \\ \rho_{1}+\left(\rho_{2}-\rho_{1}\right) \psi / \alpha, & \text { when } 0<\psi<\alpha \\ \rho_{2}, & \text { when } \alpha<\psi<\alpha+\beta\end{cases}
$$


We introduce dimensionless variables by choosing $\alpha / c$ as reference length, $c$ as reference velocity, and $\rho_{1}$ as reference density. Thus, we define the following new variables

$$
\begin{aligned}
& x^{\prime}=c x / \alpha, \quad y^{\prime}=c y / \alpha, \quad \psi^{\prime}=\psi / \alpha, \\
& \rho^{\prime}=\rho / \rho_{1}, \quad \gamma^{\prime}=\gamma / \alpha, \quad \beta^{\prime}=\beta / \alpha,
\end{aligned}
$$

and rewrite (2) in terms of the variables (3). This gives, after dropping the primes,

$$
\rho= \begin{cases}1, & \text { when }-\gamma<\psi<0 \\ 1+\left(\rho_{2}-1\right) \psi, & \text { when } 0<\psi<1 \\ \rho_{2}, & \text { when } 1<\psi<1+\beta\end{cases}
$$

The streamfunction $\psi$ satisfies Long's [4] equation. This equation can be written in terms of the function $y=f(x, \psi)$ as

$$
\frac{\rho}{2} \frac{\partial}{\partial \psi} \frac{1+f_{x}^{2}}{f_{\psi}{ }^{2}}-\rho \frac{\partial}{\partial x}\left(\frac{f_{x}}{f_{\psi}}\right)+\rho_{\psi}\left(\frac{1}{2} \frac{f_{x}{ }^{2}}{f_{\psi}{ }^{2}}+\frac{1}{2} \frac{1}{f_{\psi}{ }^{2}}-\frac{1}{2}\right)=-\lambda \rho_{\psi}(f-\psi-\gamma) .
$$

Here, $\lambda=g \alpha / c^{3}$ is a dimensionless quantity (see Vanden-Broeck and Turner [8] for a derivation of (6)). The equation (6) holds for $0<\psi<1$. For future reference we note that $f=\psi+\gamma$ is a solution of (6). This corresponds to a uniform stream with constant velocity $c$ characterized by $y=\gamma$ on $\psi=0$.

We assume that the wave is symmetric about the $y$ axis and we denote by $l$ the wavelength of the wave. Then the symmetry and periodicity of the wave gives us the boundary conditions:

$$
\begin{aligned}
& f_{x}=0, \quad 0<\psi<1, x=0, \\
& f_{x}=0, \quad 0<\psi<1, x=\frac{l}{2} .
\end{aligned}
$$

As in Vanden-Broeck and Turner [8] it may be shown that the components $\left(u_{T}(x)\right.$, $\left.v_{T}(x)\right)$ and $\left(u_{B}(x), v_{B}(x)\right)$ of the velocity on the streamlines $\psi=1$ and $\psi=0$ satisfy

$$
\begin{aligned}
u_{T}(x)- & 1-i v_{T}(x) \\
= & \frac{2}{l} \int_{0}^{\frac{l}{2}}\left[u_{T}\left(x^{\prime}\right)-1-i v_{T}\left(x^{\prime}\right)\right] \\
& \times\left[\exp \left(\frac{i 2 \pi x^{\prime}}{l}-\frac{2 \pi f\left(x^{\prime}, 1\right)}{l}\right)-\exp \left(\frac{i 2 \pi x}{l}-\frac{2 \pi f(x, 1)}{l}\right)\right]^{-1} \\
& \times \exp \left(\frac{i 2 \pi x^{\prime}}{l}-\frac{2 \pi f\left(x^{\prime}, 1\right)}{l}\right)\left(1+i \frac{\partial f\left(x^{\prime}, 1\right)}{\partial x^{\prime}}\right) d x^{\prime}
\end{aligned}
$$




$$
\begin{aligned}
& +\frac{2}{l} \int_{0}^{\frac{l}{2}}\left[u_{T}\left(x^{\prime}\right)-1+i v_{T}\left(x^{\prime}\right)\right] \\
& \times\left[\exp \left(\frac{-i 2 \pi x^{\prime}}{l}-\frac{2 \pi f\left(x^{\prime}, 1\right)}{l}\right)-\exp \left(\frac{i 2 \pi x}{l}-\frac{2 \pi f(x, 1)}{l}\right)\right]^{-1} \\
& \times \exp \left(\frac{-i 2 \pi x^{\prime}}{l}-\frac{2 \pi f\left(x^{\prime}, 1\right)}{l}\right)\left(1-i \frac{\partial f\left(x^{\prime}, 1\right)}{\partial x^{\prime}}\right) d x^{\prime} \\
& -\frac{2}{l} \int_{0}^{\frac{l}{2}}\left[u_{T}\left(x^{\prime}\right)-1+i v_{T}\left(x^{\prime}\right)\right] \\
& \times\left[\exp \left(\frac{i 2 \pi x^{\prime}}{l}+\frac{2 \pi f\left(x^{\prime}, 1\right)}{l}-\frac{4 \pi h}{l}\right)-\exp \left(\frac{i 2 \pi x}{l}-\frac{2 \pi f(x, 1)}{l}\right)\right]^{-1} \\
& \times \exp \left(\frac{i 2 \pi x^{\prime}}{l}+\frac{2 \pi f\left(x^{\prime}, 1\right)}{l}-\frac{4 \pi h}{l}\right)\left(1-i \frac{\partial f\left(x^{\prime}, 1\right)}{\partial x^{\prime}}\right) d x^{\prime} \\
& -\frac{2}{l} \int_{0}^{\frac{1}{2}}\left[u_{T}\left(x^{\prime}\right)-1-i v_{T}\left(x^{\prime}\right)\right] \\
& \times\left[\exp \left(\frac{-i 2 \pi x^{\prime}}{l}+\frac{2 \pi f\left(x^{\prime}, 1\right)}{l}-\frac{4 \pi h}{l}\right)-\exp \left(\frac{i 2 \pi x}{l}-\frac{2 \pi f(x, 1)}{l}\right)\right]^{-1} \\
& \times \exp \left(\frac{-i 2 \pi x^{\prime}}{l}+\frac{2 \pi f\left(x^{\prime}, 1\right)}{l}-\frac{4 \pi h}{l}\right)\left(1+i \frac{\partial f\left(x^{\prime}, 1\right)}{\partial x^{\prime}}\right) d x^{\prime},
\end{aligned}
$$

$$
\begin{aligned}
u_{B}(x)- & 1-i v_{B}(x) \\
= & \frac{2}{l} \int_{0}^{\frac{1}{2}}\left[u_{B}\left(x^{\prime}\right)-1-i v_{B}\left(x^{\prime}\right)\right] \\
& \times\left[\exp \left(\frac{-i 2 \pi x^{\prime}}{l}+\frac{2 \pi f\left(x^{\prime}, 0\right)}{l}\right)-\exp \left(\frac{-i 2 \pi x}{l}+\frac{2 \pi f(x, 0)}{l}\right)\right]^{-1} \\
& \times \exp \left(\frac{-i 2 \pi x^{\prime}}{l}+\frac{2 \pi f\left(x^{\prime}, 0\right)}{l}\right)\left(1+i \frac{\partial f\left(x^{\prime}, 0\right)}{\partial x^{\prime}}\right) d x^{\prime} \\
+ & \frac{2}{l} \int_{0}^{\frac{l}{2}}\left[u_{B}\left(x^{\prime}\right)-1+i v_{B}\left(x^{\prime}\right)\right] \\
& \times\left[\exp \left(\frac{i 2 \pi x^{\prime}}{l}+\frac{2 \pi f\left(x^{\prime}, 0\right)}{l}\right)-\exp \left(\frac{-i 2 \pi x}{l}+\frac{2 \pi f(x, 0)}{l}\right)\right]^{-1} \\
& \times \exp \left(\frac{i 2 \pi x^{\prime}}{l}+\frac{2 \pi f\left(x^{\prime}, 0\right)}{l}\right)\left(1-i \frac{\partial f\left(x^{\prime}, 0\right)}{\partial x^{\prime}}\right) d x^{\prime} \\
& -\frac{2}{l} \int_{0}^{\frac{1}{2}}\left[u_{B}\left(x^{\prime}\right)-1+i v_{B}\left(x^{\prime}\right)\right]
\end{aligned}
$$




$$
\begin{aligned}
& \times\left[\exp \left(\frac{-i 2 \pi x^{\prime}}{l}-\frac{2 \pi f\left(x^{\prime}, 0\right)}{l}\right)-\exp \left(\frac{-i 2 \pi x}{l}+\frac{2 \pi f(x, 0)}{l}\right)\right]^{-1} \\
& \times \exp \left(\frac{-i 2 \pi x^{\prime}}{l}-\frac{2 \pi f\left(x^{\prime}, 0\right)}{l}\right)\left(1-i \frac{\partial f\left(x^{\prime}, 0\right)}{\partial x^{\prime}}\right) d x^{\prime} \\
& -\frac{2}{l} \int_{0}^{\frac{l}{2}}\left[u_{B}\left(x^{\prime}\right)-1-i v_{B}\left(x^{\prime}\right)\right] \\
& \times\left[\exp \left(\frac{i 2 \pi x^{\prime}}{l}-\frac{2 \pi f\left(x^{\prime}, 0\right)}{l}\right)-\exp \left(\frac{-i 2 \pi x}{l}+\frac{2 \pi f(x, 0)}{l}\right)\right]^{-1} \\
& \times \exp \left(\frac{i 2 \pi x^{\prime}}{l}-\frac{2 \pi f\left(x^{\prime}, 0\right)}{l}\right)\left(1+i \frac{\partial f\left(x^{\prime}, 0\right)}{\partial x^{\prime}}\right) d x^{\prime}
\end{aligned}
$$

The parameter $h$ is the dimensionless value of $y$ on the upper wall.

On the top and bottom walls, we have the boundary conditions

$$
\begin{aligned}
& \text { the value of steamfunction } \psi \text { on the top wall }=1+\beta \text {, } \\
& \text { the value of steamfunction } \psi \text { on the bottom wall }=-\gamma \text {. }
\end{aligned}
$$

Next, by denoting the real parts of the right-hand sides of (8) and (9) by $R_{T}$ and $R_{B}$ and by using the identities

$$
\begin{aligned}
u_{T}(x) & =\left[f_{\psi}(x, 1)\right]^{-1}, \\
v_{T}(x) & =\left[f_{x}(x, 1)\right]\left[f_{\psi}(x, 1)\right]^{-1}, \\
u_{B}(x) & =\left[f_{\psi}(x, 0)\right]^{-1}, \\
v_{B}(x) & =\left[f_{x}(x, 0)\right]\left[f_{\psi}(x, 0)\right]^{-1}
\end{aligned}
$$

we obtain the integro-differential equations

$$
\begin{aligned}
& {\left[f_{\psi}(x, 1)\right]^{-1}-1=R_{T},} \\
& {\left[f_{\psi}(x, 0)\right]^{-1}-1=R_{B} .}
\end{aligned}
$$

We define the amplitude $a$ of the wave by

$$
a=f(x, 1)-1-\gamma
$$

The mathematical problem we are considering is the following: for given values of the parameters $a, \rho_{2}, h, \beta, \gamma$ and $l$, we want to solve the partial differential equation (6) in the domain

$$
0<x<\frac{l}{2}, \quad 0<\psi<1 \text {, }
$$


with the four boundary conditions (7), (10), (15) and (16). The parameter $\lambda$ is to be found as part of the solution.

The problem solved by Vanden-Broeck and Turner [8] differs from ours, because they do not use the conditions (10). Since the parameter $\beta$ appears only in (10), their scheme converges to solution for which a particular value of $\beta$ is found as part of the solution. Therefore their solutions are particular solutions of the general mathematical problem defined in the previous paragraph.

Since the Bernoulli's head was chosen such that the uniform stream is a solution of Long's equation (see remark after equation (6)), the most interesting solutions are the ones for which the value of $\beta$ is consistent with a uniform stream. Therefore we consider in this paper solutions satisfying the condition

$$
\beta=h-1-\gamma .
$$

The numerical procedure follows closely Vanden-Broeck and Turner [8]. We first introduce the mesh points

$$
\begin{aligned}
x_{I} & =[(I-1) /(2 M-2)] l, & I & =1, \ldots, M, \\
\psi_{J} & =[(J-1) /(N-1)], & J & =1, \ldots, N,
\end{aligned}
$$

and the unknowns

$$
f_{I J}=f\left(x_{l}, \psi_{J}\right), \quad I=1, \ldots, M ; J=1, \ldots, N .
$$

We satisfy the partial differential equation (6) at the mesh points $\left(x_{I}, \psi_{J}\right) ; I=$ $2, \ldots, M-1 ; J=2, \ldots, N-1$, and boundary conditions (7) at $\psi_{J}, J=1, \ldots, N$.

The boundary equations (15) and (16) are imposed at the midpoints $x^{\prime}{ }_{M}=\left(x_{I}+\right.$ $\left.x_{I+1}\right) / 2, I=1, \ldots, M-3$

The last three equations come from (10) and (17). For given values of $a, \rho_{2}, \beta$, $\gamma$ and $l$, the system of $M N+1$ equations for the $M N+1$ unknowns $\lambda, f_{l J}, I=$ $1, \ldots, M, J=1, \ldots, N$ is solved by Newton's method.

In Vanden-Broeck and Turner's [8] scheme, the equations (10) were not used and (15) and (16) were satisfied at the midpoints $x_{M}^{l}, I=1, \ldots, M-2$.

The left hand sides of $(10)$ are evaluated by first calculating $(u, v)$ in the irrotational parts of the flow in terms of the unknowns by using the Cauchy integral equation formula. We then integrate numerically $u=\psi_{y}$.

\section{Discussion of results}

Vanden-Broeck and Turner [8] presented solutions for $a=0.32, \rho_{2}=0.5, \gamma=10$ and $h=21$. We repeated their calculations and substituted their solutions into (10). 
We found that the second of the conditions (10) is approximately satisfied. For example, the values of the left hand side of the second of the conditions (10) takes the values $-10.35,-10.49,-9.9$ and -9.2 for the solutions presented in their Figures $4 \mathrm{a}-4 \mathrm{~d}$. On the other hand, substituting their solutions into the left hand side of the second of the conditions (10) gives $\beta=3.73, \beta=4.94, \beta=9.71$ and $\beta=11.79$ for their solutions in Figures 4a-4d. Therefore the solutions of Vanden-Broeck and Turner [8] do not in general satisfy (18).

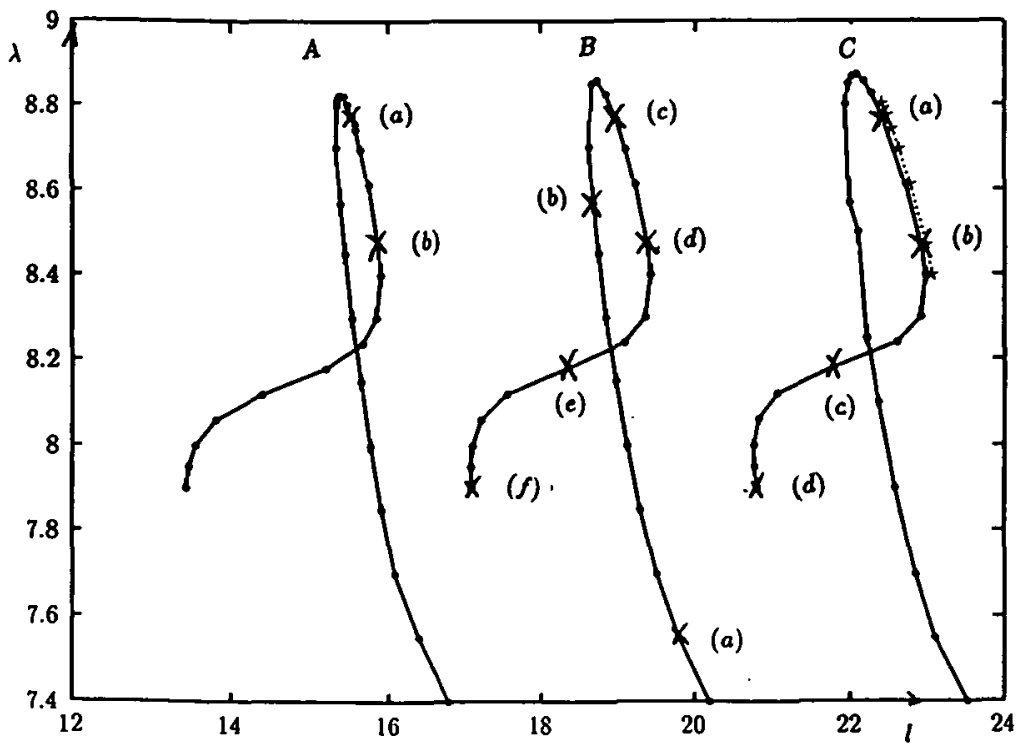

FIGURE 2. Values of $\lambda$ versus wavelength $l$ with $a=0.32, \rho_{2}=0.5, \beta=10.0, \gamma=10.0$ for three families of solutions. The broken curve was obtained by translating the portion (a)-(b) of family (A).

We used the scheme described in the previous section to compute solutions which satisfy (18). Solutions were computed for various values of $a, \rho_{2}, \beta, \gamma$. It was found that that the new solutions are qualitatively similar to those of Vanden-Broeck and Turner [8]. In particular there exist long periodic internal waves with a train of short waves in their troughs. We found that for each value of the amplitude $a$, there are many different families of solutions. Each family is characterized by the two parameters $\lambda$ and $l$. For sufficiently long waves, the families are almost parallel curves. This is illustrated in Figure 2 where we present values of $\lambda$ vs $l$ with $a=0.32, \rho_{2}=0.5$, $\beta=\gamma=10.0$ for three families. In the following discussion we denote these families by (A), (B) and (C) (see Figure 2). The families (A), (B) and (C) were computed with $N=20$ and $M=47, M=57$ and $M=67$ respectively. We checked that the results do not change within graphical accuracy when $N$ and $M$ are doubled. Typical profiles of the waves for families (B), (A) and (C) are shown in Figures 3, 4 and 5. The 20 curves on each profile correspond to the streamlines $\psi_{J}=(J-1) / 19, J=1, \ldots, 20$. 
The streamlines $\psi_{1}$ and $\psi_{20}$ separate the region of variable density from the regions of constant densities. In these Figures the Cartesian coordinates $X=x, Y=y-\gamma$ are used, and only one-half of a wavelength is shown. We note that on one wavelength, the waves of family (C) have one more wavelength of short waves in their tail than the waves of family (B) and two more wavelengths than the waves of family (A). We expect that there are an infinite number of families of solutions and therefore an infinite number of parallel curves in Figure 2. As one moves to the right from a curve to the next parallel curve, the central part of the wave remains almost unchanged and one more wavelength of short waves appears in the tail. Therefore by jumping to the right from curves to curves, we obtain in the limit a wave with an infinite oscillatory tail, that is, a generalized solitary wave.

We check these ideas by showing that the curve (C) in Figure 2 can be obtained from the curve (A), by translating the curve (A) by twice the wavelength of the short waves. Since the short waves are of small amplitude, we can use linear theory. Following Vanden-Broeck and Turner [8], we write

$$
y=f(x, \psi)=\psi+\gamma+w(x, \psi),
$$

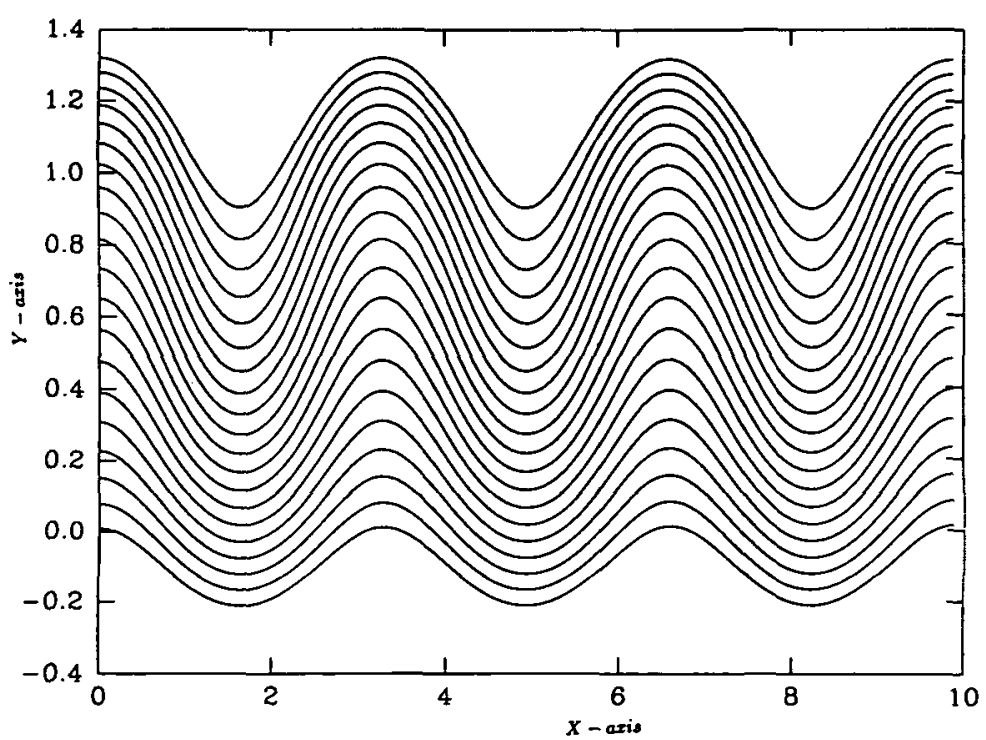

FIGURE 3A. Computed profile for a wave of family (B) with $a=0.32, \rho_{2}=0.5, \gamma=10, \lambda=7.55$. This profile corresponds to the point (a) on the family (B) of Figure 2. 


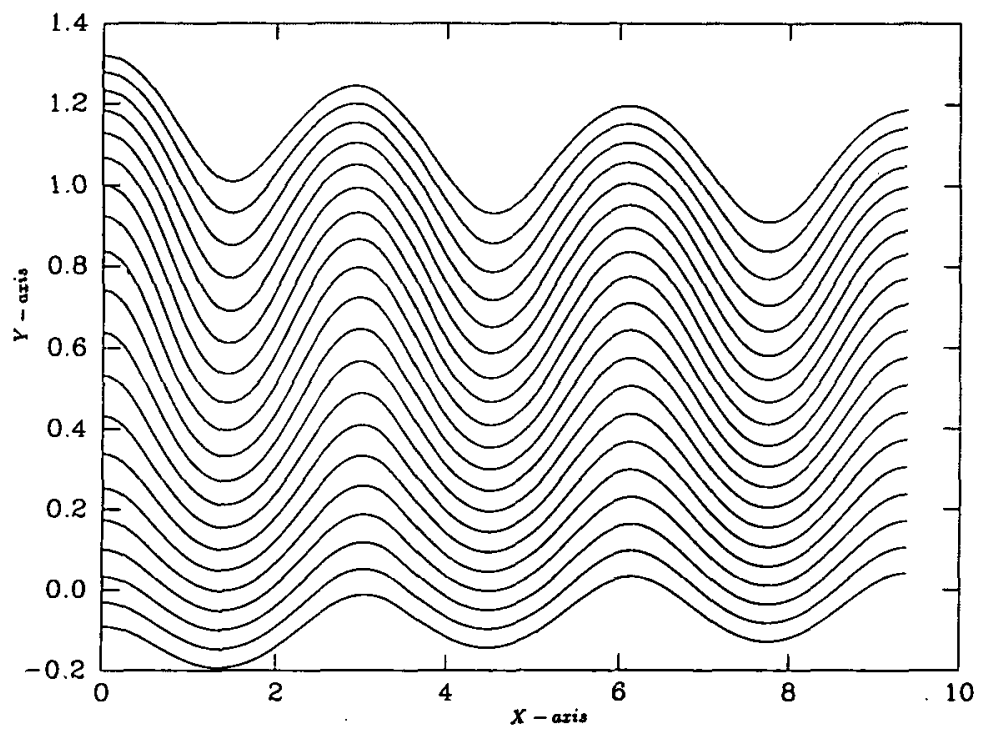

FIGURE 3B. Same as $3 \mathrm{~A}$ with $\lambda=8.568$. This profile corresponds to the point (b) on the family (B) of Figure 2 .

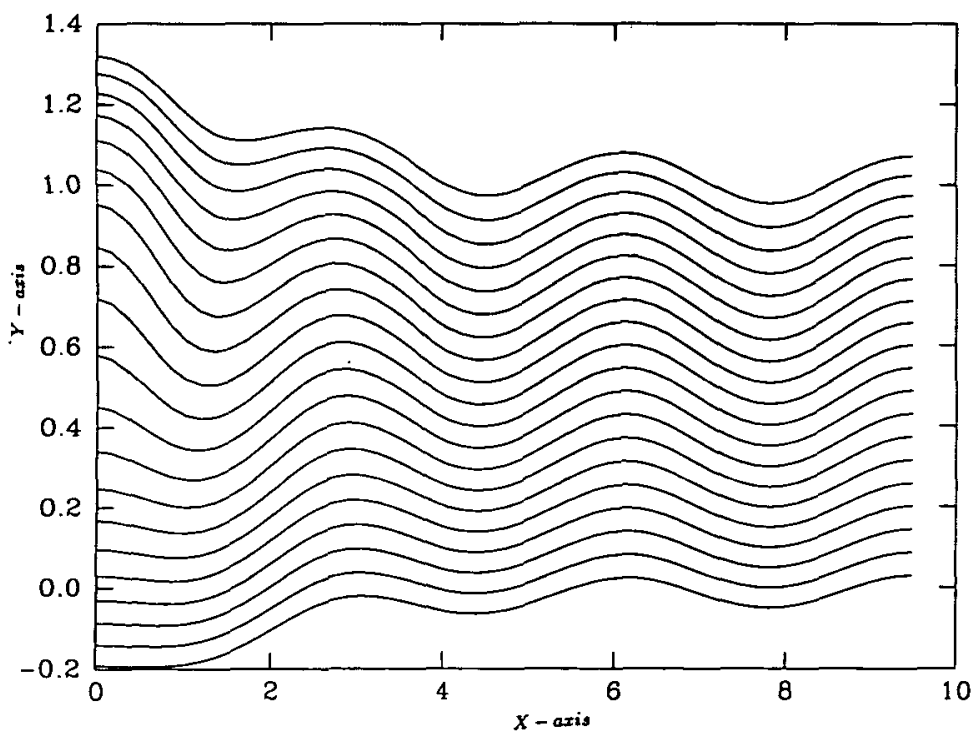

FIGURE 3C. Same as $3 \mathrm{~A}$ with $\lambda=8.765$. This profile corresponds to the point (c) on the family (B) of Figure 2. 


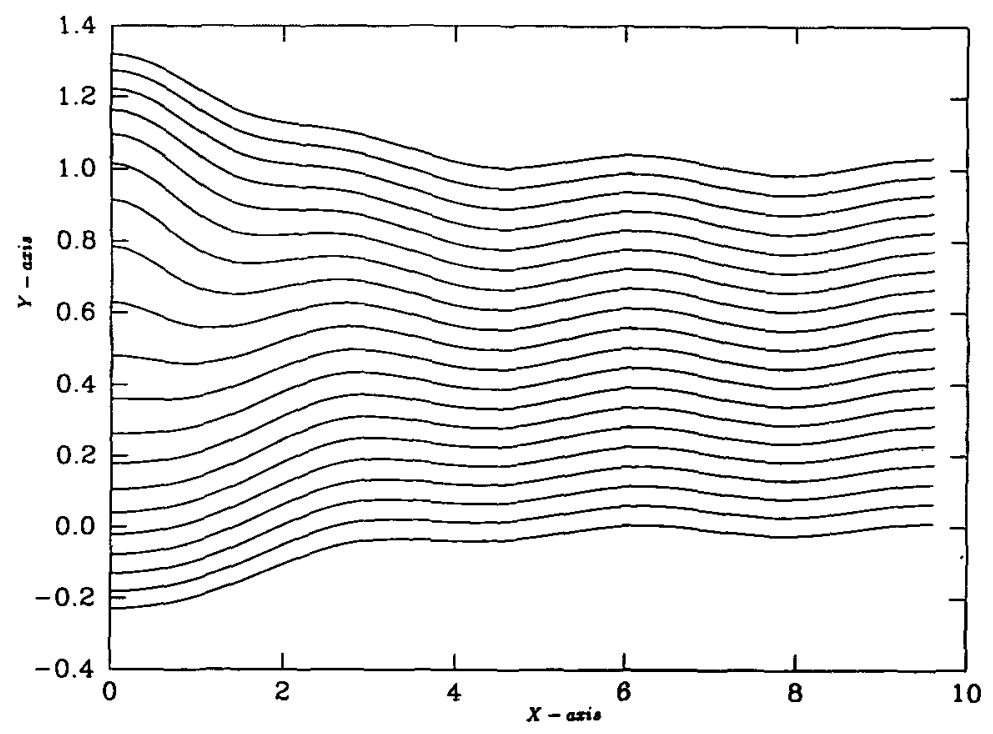

FIGURE 3D. Same as $3 \mathrm{~A}$ with $\lambda=8.47$. This profile corresponds to the point (d) on the family (B) of Figure 2.

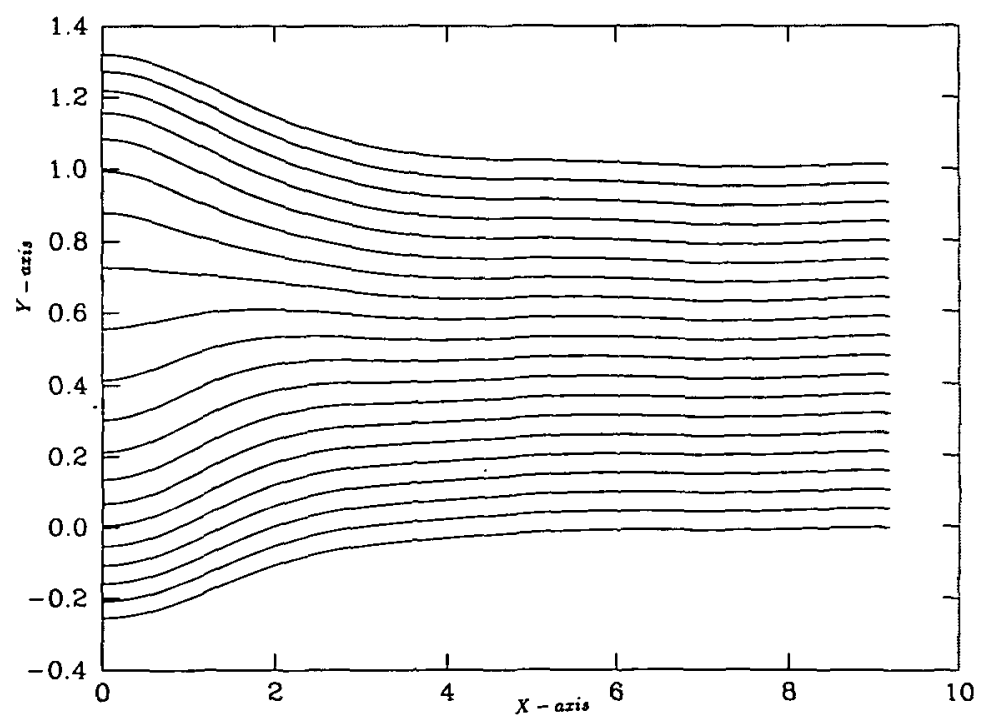

FIGURE 3E. Same as $3 \mathrm{~A}$ with $\lambda=8.18$. There are essentially no short waves in the trough. This profile corresponds to the point (e) on the family (B) of Figure 2. 


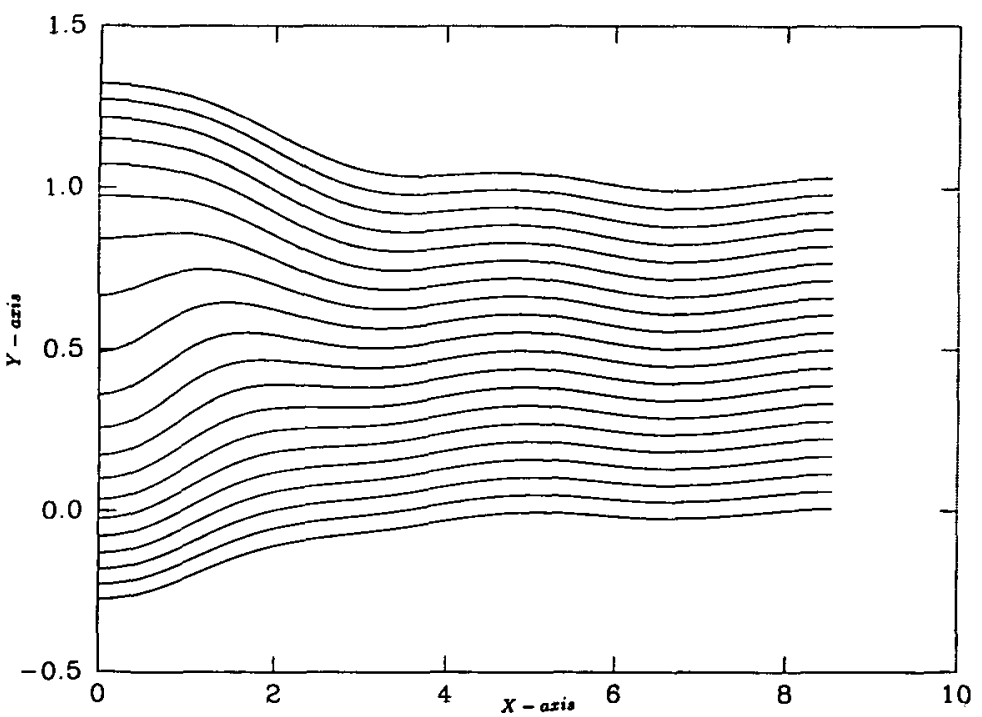

FIGURE 3F. Same as $3 \mathrm{~A}$ with $\lambda=7.9$. This profile corresponds to the point (f) on the family (B) of Figure 2.

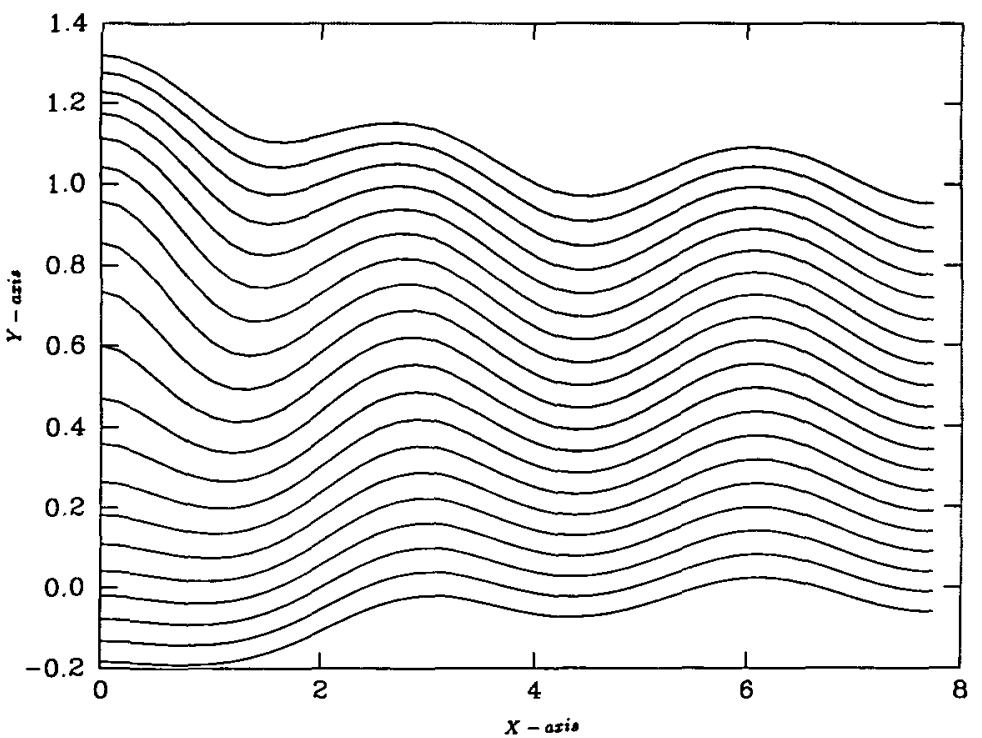

FIGURE 4A. Computed profile for family (A) with $a=0.32, \rho_{2}=0.5, \gamma=10, \lambda=8.775$. This profile corresponds to the point (a) on the family (A) of Figure 2. 


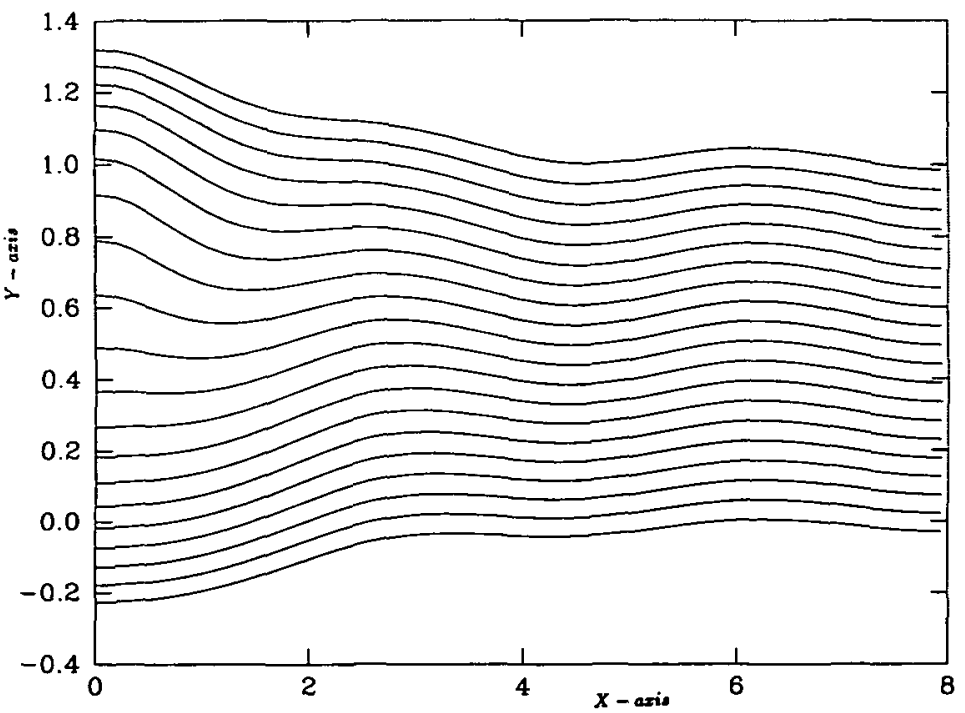

FIGURE 4B. Same as $4 \mathrm{~A}$ with $\lambda=8.47$. This profile corresponds to the point (b) on the family (A) of Figure 2.

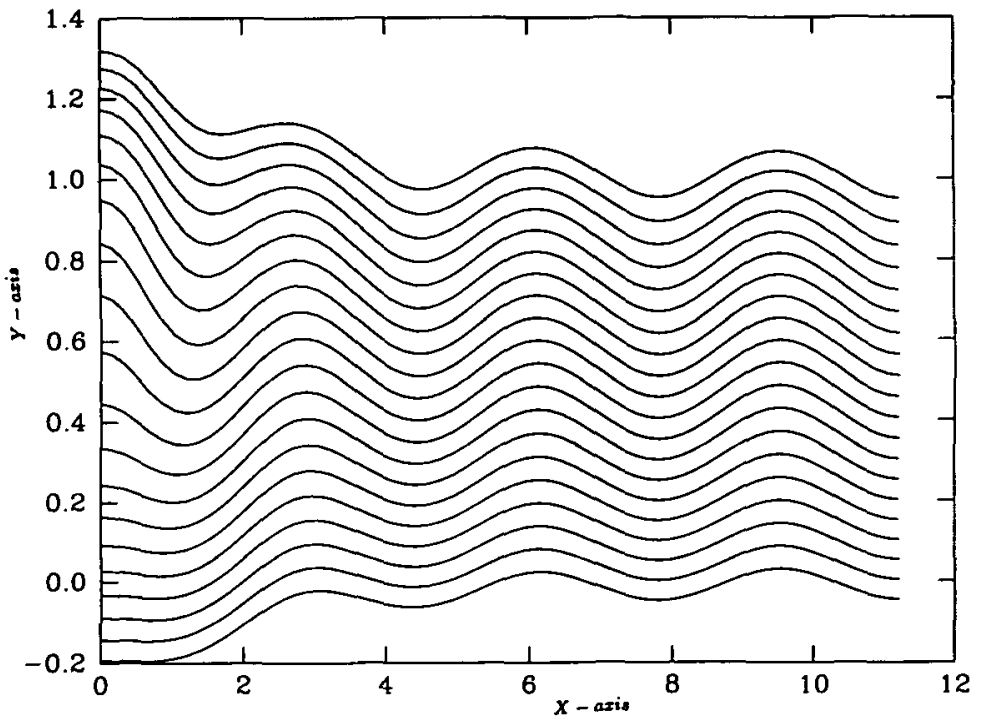

FIGURE 5A. Computed profile for family (C) with $a=0.32, \rho_{2}=0.5, \gamma=10, \lambda=8.765$. This profile corresponds to the point (a) on the family (C) of Figure 2. 


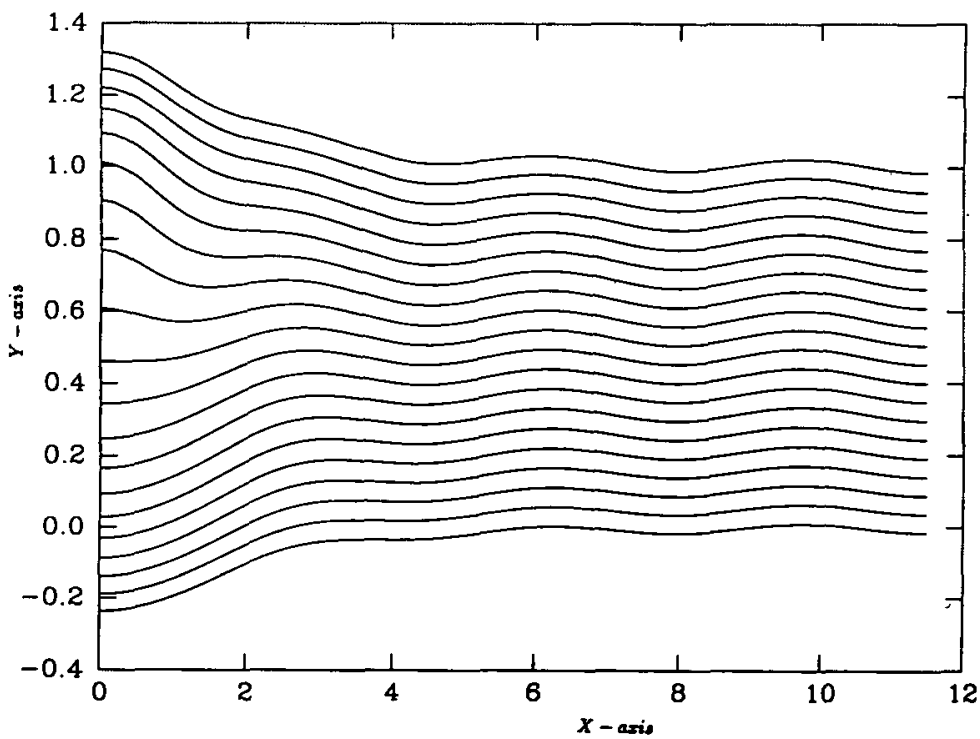

FIGURE 5B. Same as 5A with $\lambda=8.47$. This profile corresponds to the point (b) on the family (C) of Figure 2.

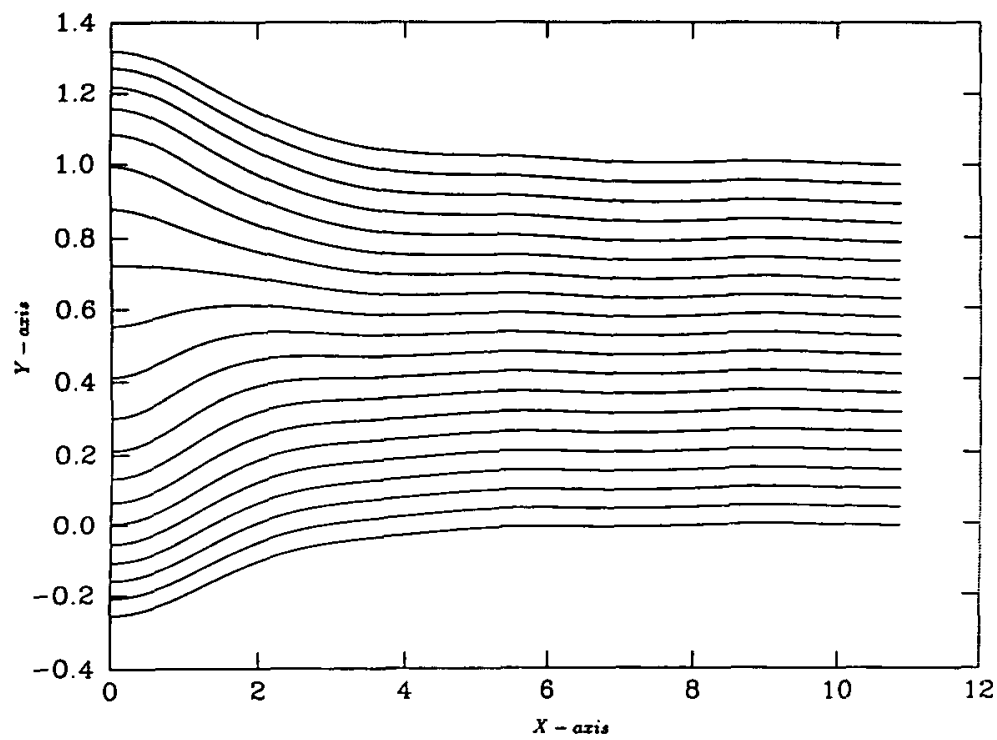

FIGURE 5C. Same as 5A with $\lambda=8.18$. There are essentially no short waves on the tail. This profile corresponds to the point (c) on the family (C) of Figure 2 . 


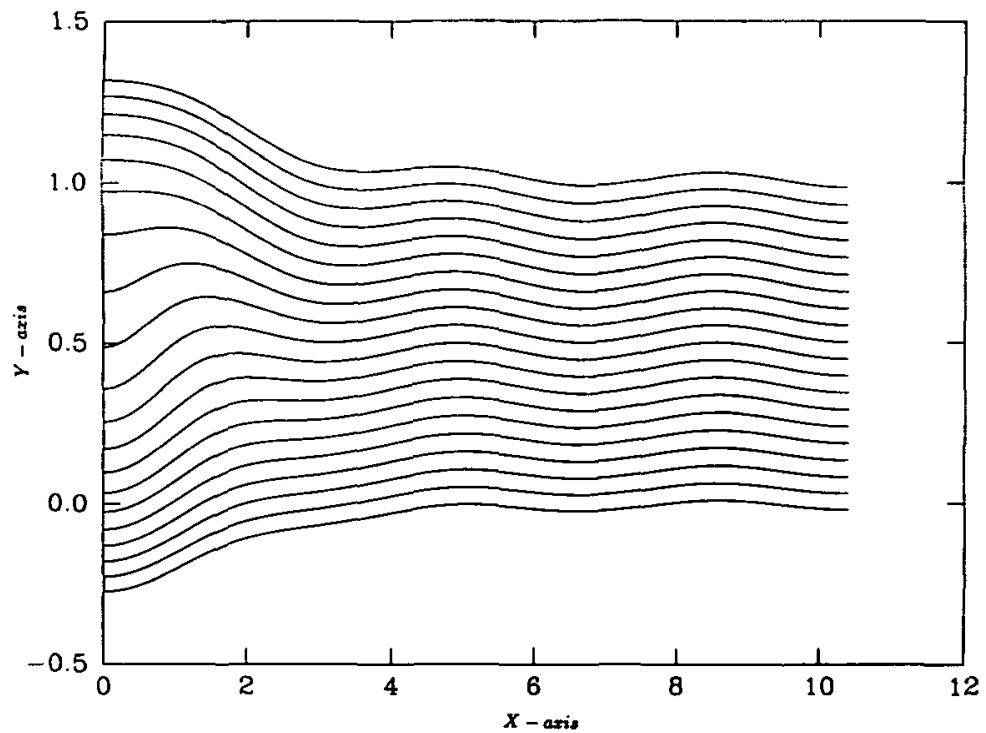

FIGURE 5D. Same as $5 \mathrm{~A}$ with $\lambda=7.9$. This profile corresponds to the point (d) on the family (C) of Figure 2.

where $w(x, \psi)$ is a small perturbation. We assume that $w(x, \psi)$ is a periodic wave satisfying $w(x, \psi)=h(\psi) \cos K x$, where $K=2 \pi / l$. We also require $w, w_{x}, w_{\psi}$ to be continuous across $\psi=0$ and $\psi=1$. Substituting (19) into the nonlinear equations of Section 2 and retaining only the terms linear in $w$, we obtain

$$
\begin{aligned}
& \left(\rho h^{\prime}\right)^{\prime}-\rho K^{2} h=\lambda \rho^{\prime} h, 0<\psi<1, \\
& h^{\prime \prime}-K^{2} h=0,-\gamma<\psi<0 \text { and } 1<\psi<1+\beta, \\
& h=0, \text { on } \psi=-\gamma \text { and } \psi=1+\beta, \\
& h\left(0_{-}\right)=h\left(0_{+}\right), \\
& h\left(1_{-}\right)=h\left(1_{+}\right), \\
& h^{\prime}\left(0_{-}\right)=h^{\prime}\left(0_{+}\right), \\
& h^{\prime}\left(1_{-}\right)=h^{\prime}\left(1_{+}\right) .
\end{aligned}
$$

For a given value of $K,(20)$ defines an eigenvalue problem with eigenvalues

$$
0<\lambda_{1}(K)<\lambda_{2}(K)<\lambda_{3}(K)<\ldots,
$$

and eigenfunctions $h_{n}(\psi), n=1,2, \ldots$ We refer to the solution $h_{n}(\psi) \cos K x$, corresponding to the eigenvalue $\lambda_{n}(K)$, as a periodic wave of mode $n$.

For each value of $\lambda$ between the points (a) and (b) on the family (A) of Figure 2, we use the system (20) to calculate the wavelength $l^{\prime}$ of small periodic waves of mode 1 . 


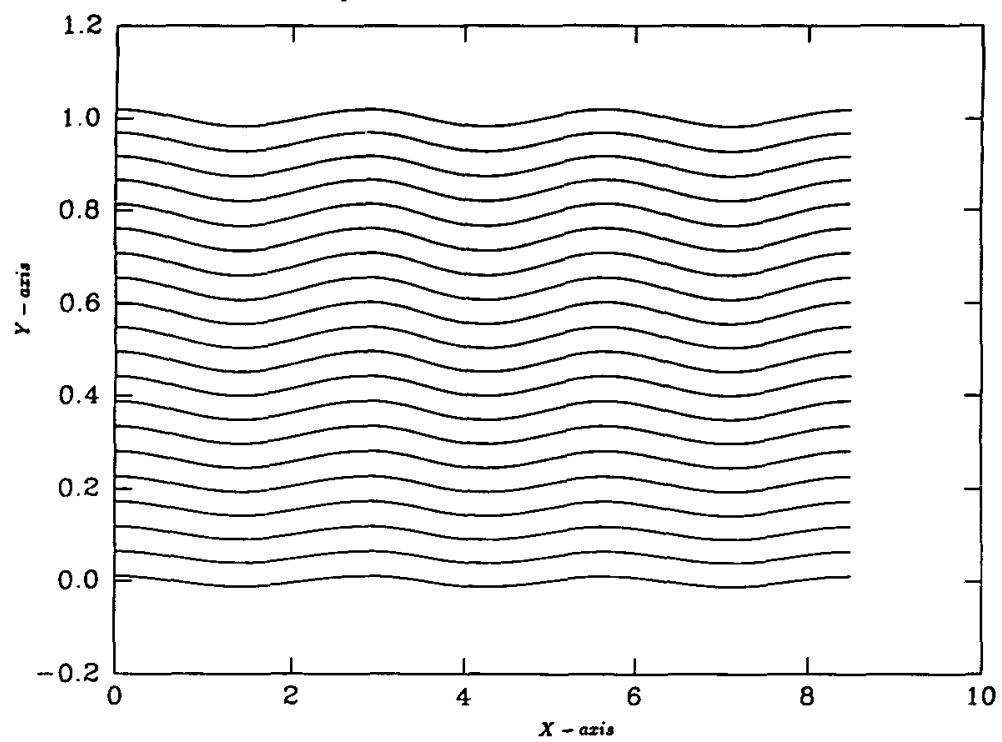

FIGURE 6. Computed profile for a wave of small amplitude with $a=0.02, \rho_{2}=0.5, \beta=10.0, \gamma=$ 10 , and $\lambda=11.6$. This solution corresponds to the cross in Figure 7.

This was done by discretizing (20) and solving the resultant algebraic equations by Newton's method. After we get the values of $l^{\prime}$, we add twice the value of $l^{\prime}$ to the wavelength $l$ of the solutions of family (A) to do the translation. This yields the broken curve of Figure 2. The almost perfect fitting between the broken curve and the (a)-(b) part of family (C) shows that the short waves in the tail of solutions are periodic waves of mode 1 . This also implies that the long periodic waves of Figures 3,4 and 5 are good approximations of generalized solitary waves. Therefore we may use the family (B) to describe the properties of generalized solitary waves.

Figure $3 \mathrm{~A}$ shows that our solitary waves bifurcate from a periodic train of waves (this is to be contrasted with the solutions of Vanden-Broeck and Turner [8] which bifurcate from conjugate flows). As we move along the branch the main crest emerges from the train of periodic waves and we obtain a generalized solitary wave. The amplitude of the short waves first decreases and then increases. At some point along the curve, the amplitude of the short waves is essentially zero and the generalized solitary wave becomes very close to a familiar solitary wave (see Figure $3 E$ ). Such generalized solitary waves with essentially no short waves in the trough were previously found for surface waves with surface tension (see [7]).

We also calculated internal waves of small amplitude for $a=0.02, \rho_{2}=0.5, \beta=$ $\gamma=10.0$. One typical profile is shown in Figure 6. The values of $\lambda$ vs $l$ are shown in Figure 7. The curve ultimately terminates around $\lambda=14.36$. We also found that when the amplitude is small, the wave tends to be sine-like. The dotted line in Figure 7 


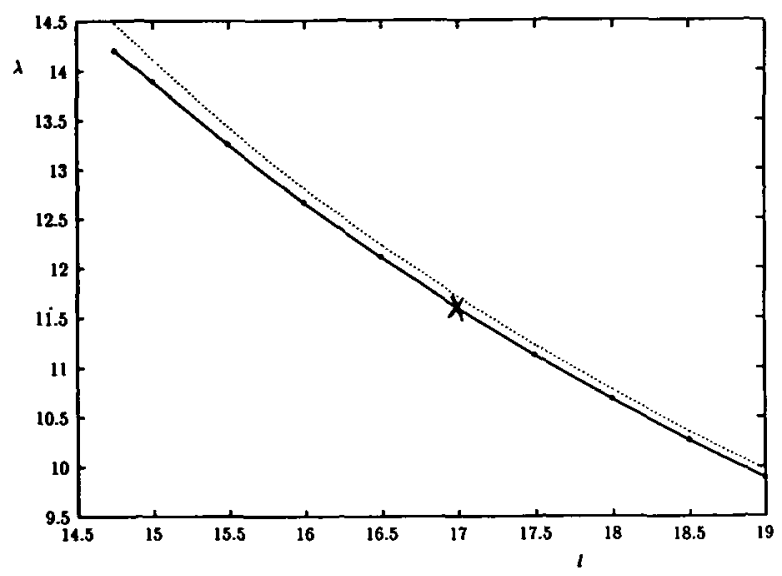

FIGURE 7. Values of $\lambda$ versus the wavelength $l$ with $a=0.02, \rho_{2}=0.5, \beta=10.0, \gamma=10.0$ for one family of solutions. The dotted line was computed from (20).

is calculated from (20). The fitting between them constitutes a check on the accuracy of our scheme.

\section{Acknowledgment}

This work was supported in part by the National Science Foundation and the Lady Davis Foundation. The second author gratefully acknowledges the hospitality of the Hebrew University of Jerusalem, Tel Aviv University and the Technion (Haifa).

\section{References}

[1] T. R. Akylas and R. H. J. Grimshaw, "Solitary internal waves with oscillatory tails", J. Fluid Mech. 242 (1992) 279-298.

[2] J. T. Beale, "Exact solitary water waves with capillary ripples at infinity", Commun. Pure Appl. Math. 44 (1991) 211-257.

[3] J. K. Hunter and J.-M. Vanden-Broeck, "Solitary and periodic gravity-capillary waves of finite amplitude", J. Fluid Mech. 134 (1982) 205-219.

[4] R. R. Long, "Some aspects of the flow of stratified fluids", Tellus 5 (1953) 42-58.

[5] S. M. Sun, "Existence of a generalized solitary wave solution for water with positive Bond number smaller than 1/3", J. Math. Anal. Appl. 156 (1991) 471-504.

[6] S. M. Sun and M. C. Shen, "Exponentially small asymptotics for internal solitary waves with oscillatory tails in a stratified fluid", Math. Appl. Anal. 1 (1994) 81-107. 
[7] J.-M. Vanden-Broeck, "Elevation solitary waves with surface tension", Phys. Fluids A 3 (1991) 2659-2663.

[8] J.-M. Vanden-Broeck and R. E. L. Turner, "Long periodic internal waves", Phys. Fluids A 4 (1992) 1929-1935. 